\title{
Las oil bottles fenicias en la península ibérica: novedades y perspectivas de la investigación
}

\section{Phoenician "oil bottles" in the Iberian Peninsula: update and research outlooks}

\author{
FRANCISCO B. GOMES \\ UNIARQ - Centro de Arqueologia da Universidade de Lisboa, Faculdade de Letras da Universidade de Lisboa, \\ Fundação para a Ciência e Tecnologia \\ Alameda da Universidade, 1600-214, Lisboa, Portugal \\ franciscojbgomes@gmail.com
}

El corpus de los pequeños contenedores fenicios para perfumes usualmente designados oil bottles de la península ibérica, ampliado en los últimos años por numerosos hallazgos reseñados en este trabajo, permite hoy establecer una aproximación a la evolución diacrónica de la difusión de estas piezas y de sus contenidos y bien así una primera valoración de las lógicas de aprovisionamiento y de consumo de los mismos. Ese análisis permite además situar estas oil bottles en el marco más amplio del comercio de perfumes mediterráneos en la Edad del Hierro peninsular.

\section{PALABRAS CLAVE}

OIL BOTTLES, COMERCIO FENICIO, PERFUMES, CONSUMO, I EDAD DEL HIERRO, PENÍNSULA IBÉRICA

The corpus of the small Phoenician perfume containers usually known as "oil bottles" from the Iberian Peninsula, enlarged in recent years by numerous finds which are compiled in this work, can serve as the basis for an approach to the diachronic evolution of the diffusion of these pieces and their contents as well as for a first consideration of the dynamics of supply and consumption of these products. Such an analysis helps situate these "oil bottles" in the larger framework of the trade of Mediterranean perfumes in the Iberian Iron Age.

\section{KEYWORDS}

OIL BOTTLES, PHOENICIAN TRADE, PERFUMES, CONSUMPTION, EARLY IRON AGE, IBERIAN PENINSULA 


\section{La investigación sobre las oil bottles fenicias en la península ibérica: breve repaso historiográfico}

Desde la publicación en los años setenta de los estudios pioneros de A. M. ${ }^{a}$ Bisi (1970: 31) y W. Culican (1970), las llamadas oil bottles fenicias, ocasionalmente referenciadas en la literatura hispana como ampollas, se han convertido en un indicador privilegiado de la presencia y del comercio fenicio a lo largo del Mediterráneo. En este proceso, la documentación de la península ibérica ha tenido un papel importante.

A los primeros datos aportados por las excavaciones realizadas en los asentamientos coloniales fenicios de la costa oriental andaluza a partir de los años sesenta, habría que sumar a inicios de los ochenta la publicación de los estudios fundamentales de A. González Prats (1982) y J. Ramon Torres (1982). Estos trabajos han contribuido de manera decisiva a impulsar la investigación sobre esta categoría de contenedores, situándolos además claramente en el ámbito del comercio fenicio de perfumes y sustancias aromáticas.

En años posteriores, los trabajos en los núcleos fenicios del sur peninsular han aportado un ingente volumen de datos sobre la circulación e incluso la producción ( $c f$. Martín Córdoba et al., 2006) de estos contenedores, mientras que la investigación en los yacimientos «orientalizantes» ha arrojado importantes datos sobre la difusión de estos contenedores en ambientes indígenas (v. Orsingher, 2010, con bibliografía exhaustiva).

Sin embargo, dicha información, dispersa en distintas publicaciones, no se sistematizó hasta fechas más recientes. De hecho, esta labor solo se ha materializado en una importante síntesis publicada por A. Orsingher (2010) a inicios de esta década, donde, en el marco de su exhaustivo análisis de la difusión de estos contenedores en el Mediterráneo, el autor realiza por primera vez una recopilación de toda la documentación peninsular publicada hasta la fecha (Orsingher, 2010: 47-52).

Dicho trabajo sigue siendo insuperable por su exhaustividad y por el volumen de datos que recoge. Sin embargo, en los últimos años se han producido varios avances importantes con respecto al conocimiento del mundo colonial fenicio en la península ibérica y al desarrollo de las comunidades locales peninsulares en contacto con dicho mundo. Entre ellos, cabría mencionar el hallazgo y/o publicación de cierto número de ejemplares de oil bottles, algunos en contextos muy interesantes para la discusión global de este grupo de contenedores, de su difusión y, sobre todo, de su consumo en el ámbito de la península ibérica.

La presente contribución pretende sistematizar esos nuevos hallazgos, poniendo al día el catálogo de las oil bottles peninsulares realizado en su día por Orsingher (2010) y discutiendo el significado de los hallazgos más recientes. Por su parte, en la sección final se ofrecen algunas reflexiones preliminares sobre el papel de estos contenedores en el marco del consumo de perfumes mediterráneos en la Edad del Hierro de la península ibérica, que constituyen un adelanto de un proyecto más amplio sobre este tema actualmente en curso. 


\section{Oil bottles fenicias en la península ibérica: novedades de la investigación}

\subsection{La introducción y la distribución de las oil bottles fenicias en Occidente: novedades del ámbito colonial fenicio}

\subsubsection{El ámbito atlántico: Cádiz y Ayamonte}

En la recopilación realizada por Orsingher (2010: 47-52), un $76 \%$ de las oil bottles peninsulares citadas provenía de ambientes coloniales fenicios. Por ello, no resulta sorprendente que el mayor número de nuevos hallazgos acaecidos en los últimos años también se haya producido en ese ámbito colonial.

Particularmente interesante en este contexto es el caso de Cádiz, la antigua metrópolis colonial fenicia de Gadir. En el momento en que Orsingher produjo su síntesis, y a pesar de las abundantísimas excavaciones realizadas en el casco urbano de la ciudad, las únicas oil bottles conocidas en este importante yacimiento eran los dos ejemplares procedentes de la excavación del solar 38 de la calle Cánovas del Castillo (Córdoba Alonso y Ruiz Mata, 2005: 1283-1287 y fig. 8); considerados la importancia de este centro colonial y el volumen de intervenciones allí realizadas, esta situación resultaba un tanto anómala.

Sin embargo, la reciente publicación de un volumen dedicado a los últimos trabajos efectuados en el área urbana de Cádiz (Botto, 2014) ha cambiado sustancialmente la situación, ya que en ella se da a conocer un número significativo de nuevas oil bottles provenientes de distintas intervenciones.

Algunos de los datos más interesantes se han recuperado en la excavación desarrollada en el solar del antiguo Teatro Cómico de la ciudad (Gener Basallote et al., 2014; Torres Ortiz et al., 2014). Entre los materiales recuperados en la importante secuencia fenicia arcaica aquí documentada se cuenta un conjunto sustancial de contenedores del tipo en cuestión que añade datos interesantes de cara a la valoración de los parámetros cronológicos y productivos de estos envases.

Las oil bottles más antiguas recogidas en esta intervención provienen de contextos relacionados con la etapa final del llamado Período II - Fenicio A, entre 780 y 760 a.n.e. (Torres Ortiz et al., 2014: 60-61; cf. tb. Gener Basallote et al., 2014: 18-37). Entre los materiales atribuidos a este horizonte se cuentan cuatro oil bottles, tres de ellas completas, mientras de la cuarta solo se conserva la porción superior (Torres Ortiz et al., 2014: 60-61 y fig. 7) (fig. 1, n. ${ }^{\text {os }} 1-4$ ).

Por su morfología, caracterizada por cuerpos globulares y fondos planos con un incipiente pie de anillo, estas piezas se encuadran de manera clara en las series más antiguas de esta tipología cerámica (Torres Ortiz et al., 2014: 60; cf. Orsingher, 2010: tab. I), como cabría esperar dado su contexto estratigráfico.

Aunque en el avance del estudio de los materiales de la excavación del Teatro Cómico no se adelanten las características productivas de estas piezas, se ha considerado que por 


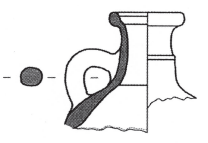

1

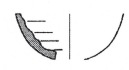

5

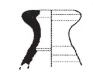

9

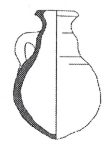

13

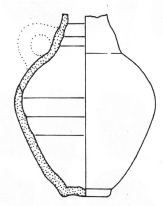

17

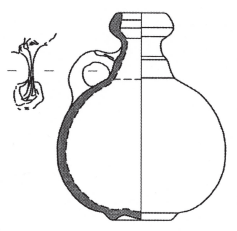

2

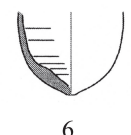

-

10

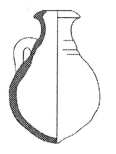

14

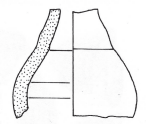

18

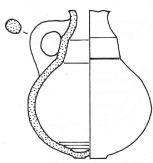

21

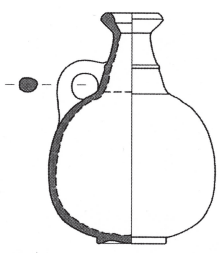

3

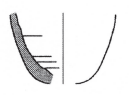

7

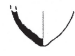

11

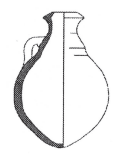

15

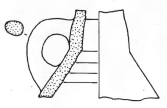

19

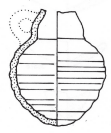

22

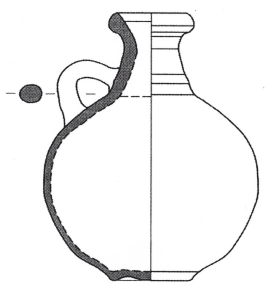

4

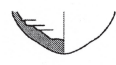

8

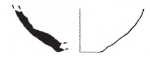

12

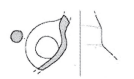

16

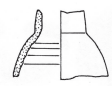

20

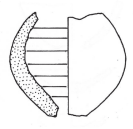

23

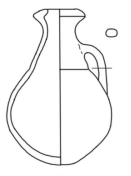

24

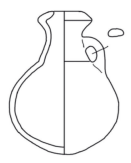

25

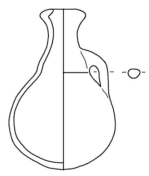

26

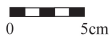

Figura 1. Oil bottles procedentes de contextos fenicios (hallazgos recientes): 1-8. Teatro Cómico de Cádiz (seg. Torres Ortiz et al., 2014); 9-12. Castillo de San Sebastián (Cádiz) (seg. Maya Torcelly et al., 2014); 13-15. Bodegas Abarzurza (Cádiz) (seg. Belizón Aragón et al., 2014); 16. La Caleta (Cádiz) (seg. Sáez Romero e Higueras-Milano Castellano, 2016); 17-23. La Fonteta (Alicante) (seg. González Prats, 2014b); 24-26. Bajo de la Campana (Cartagena) (seg. Belizón Aragón et al., 2014). 
sus características podrían corresponder a producciones orientales (Belizón Aragón et al., 2014: 212); de confirmarse, este dato tendría particular interés para la discusión de las dinámicas de introducción de estos contenedores -y de sus contenidos- en Occidente.

De todos modos, estas piezas se incluyen entre las oil bottles fenicias más antiguas documentadas hasta la fecha en territorio peninsular, sumándose a los ya citados ejemplares de la calle Cánovas del Castillo (Córdoba Alonso y Ruiz Mata, 2005: 1283-1287, fig. 8) y a los de la fase Bl del Morro de Mezquitilla, en Vélez-Málaga (Schubart, 1985: 160, fig. 9, g; Maass-Lindemann, 1999: 130-131, fig. 3), hasta el momento los únicos conocidos con fechas seguramente de la primera mitad del siglo vIII a.n.e.

En la fase siguiente del yacimiento, Período III-Fenicio B (mediados del siglo vIII a inicios del vi a.n.e.) (cf. Gener Basallote et al., 2014: 37-41), también se han documentado ejemplares de oil bottles: se han publicado cuatro ejemplares representados por sendos fondos que evidencian la presencia de piezas de cuerpo piriforme y fondo en punta (Torres Ortiz et al., 2014: 66-69, fig. 15, j-ll) (fig. 1, n. ${ }^{\text {os }}$ 5-8).

En este caso sí se hacen algunas puntualizaciones con respecto a las características productivas de estos contenedores, como que los mismos se reparten entre producciones orientales y del Mediterráneo central, en concreto del área de Cartago (Torres Ortiz et al., 2014: 69). Los datos del yacimiento gaditano demuestran que no solo las oil bottles de la serie más antigua se produjeron en el o los talleres orientales, ya que en este caso se identifican con claridad ejemplares de morfologías relativamente evolucionadas procedentes de esos mismos talleres (cf. Belizón Aragón et al., 2014: 211-212).

Otra excavación publicada en el volumen antes comentado y en la que también se han identificado varios ejemplares de oil bottles tuvo lugar en el interior del Castillo de San Sebastián, tradicionalmente identificado por la historiografía como el probable solar del santuario gaditano de Kronos mencionado en las fuentes clásicas (Maya Torcelly et al., 2014).

Esta intervención ha permitido documentar por primera vez en la pequeña isla niveles fenicios arcaicos fechados por los responsables de su estudio entre finales del siglo vII e inicios del vi a.n.e. (Maya Torcelly et al., 2014: 164-175). En dichos niveles se han recogido cuatro contenedores del tipo que nos ocupa, representados por un borde, un cuello y dos fondos, uno de ellos plano y posiblemente correspondiente a un elemento residual (Maya Torcelly et al., 2014: 171-172 y fig. 20, a-b y e-f) (fig. 1, n. ${ }^{\text {os }}$ 9-12). Entre estas piezas se han vuelto a identificar producciones orientales que permiten confirmar la situación ya comentada al respecto de los ejemplares del Período III de la excavación del Teatro Cómico (cf. supra), además de producciones centromediterráneas y posiblemente locales (Maya Torcelly et al., 2014: 171).

Aparte de la información cronológica y productiva, estos ejemplares del Castillo de San Sebastián son también muy interesantes por su posible, por no decir probable, asociación a una estructura de naturaleza religiosa.

Igual de interesantes por su contexto funcional resultan las piezas recogidas en las excavaciones del solar de las Bodegas de Abarzurza, también en la ciudad de Cádiz (Belizón Aragón et al., 2014). En esta excavación se ha identificado un conjunto funerario de cronología fenicia arcaica en el que destaca, con respecto al tema de esta contribución, la tumba 110, en la que 
se han recogido tres oil bottles completas (Belizón Aragón et al., 2014: 210-216, fig. 14) (fig. 1, n. ${ }^{\text {os }}$ 13-15). Estos tres ejemplares — con bordes reentrantes en «T», cuerpos de morfología piriforme incipiente y fondos convexos, en punta (Belizón Aragón et al., 2014) — han motivado una interesante reflexión global sobre este tipo de contenedores y sus centros de producción, orientada sobre todo hacia la demostración de la existencia de centros productores sardos a los que se asignan estos ejemplares de la necrópolis gaditana, relacionados específicamente con un taller de Bithia (Belizón Aragón et al., 2014: 215; v. tb. Ramon Torres, 1982).

Más allá de esta interesante reflexión, que aporta un dato de gran interés a la discusión ya citada sobre la diversidad de los centros productores de sustancias aromáticas, habría que subrayar la naturaleza funeraria de estos hallazgos. De hecho, y como bien señalan los responsables de su estudio (Belizón Aragón et al., 2014: 213), la presencia de oil bottles en contextos funerarios fenicios en el territorio peninsular está lejos de ser la norma. Dicha presencia solo se documenta en esta tumba y en la también recientemente publicada tumba 2 de Ayamonte (Huelva) (García Teyssandier y Marzoli, 2013; v. infra), además de en la necrópolis de Puig des Molins, en Ibiza (cf. Orsingher, 2010: 50-51, con bibliografía), lo cual contrasta con su frecuente presencia en ambientes funerarios indígenas (v. infra).

Al margen de estos tres contextos, debe citarse otra excavación también publicada en el volumen comentado: la intervención del solar 29 de la calle Ancha de Cádiz (Ruiz Mata et al., 2014).

En este trabajo se presenta una tabla en la que se compara la composición del conjunto cerámico de esta excavación con el de la intervención de la calle Cánovas del Castillo, en la que se refiere la existencia de una oil bottle también en este sector de la ciudad (Ruiz Mata et al., 2014: 107); sin embargo, si este registro corresponde a la posible "ampolla» referida e ilustrada en otros pasos del mismo trabajo (Ruiz Mata et al., 2014: 101 y fig. 13, 6), cabría tomar este dato con cautela, ya que la pieza ilustrada parece pertenecer más bien al grupo de los dippers.

Por último, a estas 15 piezas publicadas en el volumen coordinado por M. Botto habría que añadir otro ejemplar de publicación más reciente. Esta pieza, recogida en el contexto de trabajos de arqueología subacuática desarrollados en el área de La Caleta, proviene de la zona del Bajo de Chapitel (Saéz Romero e Higueras-Milena Castellano, 2016: 134-135, fig. 6, BCH/CA08/04) y se encuentra representada por un fragmento del cuello y del arranque de la panza (fig. 1, n. ${ }^{\circ}$ 16). A pesar de su estado fragmentario y falta de contexto, cabría subrayar que esta pieza se ha identificado como una producción oriental (Saéz Romero e Higueras-Milena Castellano, 2016: 134), al igual que algunos de los ejemplares ya comentados. Por otra parte, a pesar de su difícil contextualización, debe señalarse que en el marco de estas acciones de reconocimiento se ha recuperado en esta misma zona un número sustancial de dipper juglets y tres quemadores de doble cazoleta (Saéz Romero e Higueras-Milena Castellano, 2016:133-135 y fig. 6), configurando un ambiente muy particular, de posible naturaleza religiosa (Saéz Romero e Higueras-Milena Castellano, 2016: 135-137).

Además se ha planteado en múltiples ocasiones una función ritual de cara a la interpretación de los abundantes elementos excepcionales recogidos en esta zona a lo largo de las 
décadas y que se han querido relacionar con la existencia en la vecina Punta del Nao de un santuario dedicado a Astarté - Venus Marina (Saéz Romero e Higueras-Milena Castellano, 2016).

Finalmente, y antes de abandonar la bahía de Cádiz, no puede dejar de hacerse una rápida referencia a un trabajo dedicado al cerro del Castillo de Chiclana (Chiclana de la Frontera) publicado en el volumen antes citado (Bueno Serrano, 2014) en el que se refiere de modo muy breve la existencia entre los materiales cerámicos allí recogidos de "... algunos fragmentos de jarritas, ampollas y jarros de boca de seta..." (Bueno Serrano, 2014: 242). Es muy probable que entre estos se cuente algún ejemplar del tipo que aquí nos ocupa, aunque no se conozcan más detalles al respecto.

Aparte de las novedades referidas a Gadir y a su entorno inmediato, el área atlántica peninsular ha proporcionado en los últimos años otro hallazgo de gran interés para el tema de esta contribución. Me refiero a una oil bottle recogida en el fondo del pozo funerario de la tumba 2 de la necrópolis de Ayamonte, identificada por las responsables de su estudio como una producción centromediterránea (García Teyssandier y Marzoli, 2013: 106, fig. 10, d). Esta tumba, que acogió la incineración de un varón adulto, se ha fechado en el último tercio del siglo viII a.n.e. (García Teyssandier y Marzoli, 2013: 106), por lo que esta oil bottle se integra igualmente en una etapa antigua de la difusión de estos contenedores.

Sumada a los ejemplares de la tumba 110 del solar de las Bodegas Abarzurza antes comentados, esta pieza de Ayamonte permite superar el anterior desconocimiento de contenedores de este tipo en los contextos funerarios fenicios peninsulares (con la excepción de Ibiza), revelando así unas pautas de consumo más diversas dentro del propio marco colonial (v. infra).

\subsubsection{El ámbito mediterráneo: La Fonteta y Bajo de la Campana}

En el ámbito del litoral mediterráneo peninsular, las novedades relacionadas con el estudio de las oil bottle fenicias son menos numerosas, si bien la publicación definitiva de las excavaciones dirigidas por A. González Prats (2014a) en la colonia fenicia de La Fonteta (Alicante) ha aportado datos de gran interés de cara a la temática de esta contribución.

De hecho, la publicación integral de las oil bottle recogidas en este yacimiento ha incrementado sustancialmente el número de contenedores antes conocido, pasando de los dos ejemplares reseñados por A. Orsingher (2010: 50, con bibliografía) a un número máximo de 39 piezas (González Prats, 2014b: 423) (fig. 1, n. ${ }^{\text {os }}$ 17-23).

Este número - al que deben sumarse los cuatro ejemplares recogidos en las intervenciones del equipo francés que trabajó en el mismo yacimiento (Rouillard et al., 2007: fig. 181, n. ${ }^{\circ}$ 6; fig. 206, n. ${ }^{\circ} 31$; fig. 219 , n. ${ }^{\circ} 2$; fig. 237, n. ${ }^{\circ} 10$ - convierte La Fonteta en el yacimiento peninsular con el mayor número de oil bottle documentadas, muy por encima de la isla de Ibiza, con 24 ejemplares, y de Cádiz, con 18. ${ }^{1}$ Este hecho hace del enclave

1. Sin contar con el centro alfarero de la Pancha, donde al parecer se produjeron contenedores del tipo que nos ocupa y en el que se recogieron 26 ejemplares (Martín Córdoba et al., 2006: 271). 
colonial alicantino uno de los principales nudos de consumo y quizá de distribución de sustancias aromáticas del tipo contenido en estos envases.

Más allá de su número, la publicación de las oil bottle de este sector de La Fonteta ofrece otros datos de interés; desde un punto de vista productivo, entre los materiales del yacimiento se han podido identificar piezas procedentes de talleres de Andalucía Oriental (18 ejemplares), del Mediterráneo oriental (once ejemplares) y del Mediterráneo central, más específicamente del área de Cartago (cuatro ejemplares), además de ejemplares no atribuidos a ningún taller (seis) (González Prats, 2014b: 424, tabla 1).

$\mathrm{Al}$ haberse recuperado a lo largo de una secuencia estratigráfica bien caracterizada, estas piezas permiten además averiguar la representatividad de los varios grupos productivos a lo largo del período comprendido entre al menos la fase Fonteta II (720-670 a.n.e.) y la fase Fonteta VI (580-560 a.n.e.). ${ }^{2}$

A pesar de las limitaciones de la muestra, sobre todo para las fases III y VI (los estratos de las fases IV y V no han aportado ningún ejemplar), cabría resaltar que un análisis de la evolución de dicha representatividad revela, por un lado, el precoz desarrollo de las producciones peninsulares, predominantes ya en la fase II (finales del siglo vIII-inicios del vII a.n.e.) y, por otro, un posible retroceso de las importaciones orientales a partir del siglo vII a.n.e., aunque este dato no pueda considerarse totalmente demostrado. Las producciones cartaginesas, por su parte, están asimismo documentadas tanto en la fase II como en la VI (González Prats, 2014b).

Como ya se ha comentado, más allá de los nuevos datos de La Fonteta las novedades sobre la difusión de las oil bottle en el ámbito colonial fenicio de la costa mediterránea española son muy escasas. Solo cabría señalar la publicación de tres ejemplares procedentes del pecio fenicio de Bajo de la Campana (Cartagena) (Mederos Martín y Ruiz Cabrero, 2004; Polzer, 2014) cuyas ilustraciones se presentan en el marco de la ya comentada síntesis producida a propósito de las piezas de las Bodegas Abarzurza de Cádiz (Belizón Aragón et al., 2014: fig. 21, n. ${ }^{\text {os }}$ 7-9).

La presencia de oil bottles en este pecio ya había sido señalada anteriormente (Orsingher, 2010: 56), aunque no se conociera el perfil de ninguna de las piezas allí recogidas hasta la publicación de este estudio, y permite confirmar que dichas piezas presentan una morfología totalmente compatible con la cronología atribuida a este pecio (finales del siglo vII a.n.e.), al contar con los típicos cuerpos piriformes y con fondos en punta (fig. 1, n. ${ }^{\text {os }} 24-26$ ).

Aparte, aunque estas piezas se hayan considerado un posible suplemento de una carga compuesta principalmente por contenedores anfóricos (Orsingher, 2010) y por lingotes metálicos (Mederos Martín y Ruiz Cabrero, 2004; Polzer, 2014), la publicación de un conjunto importante de elementos excepcionales recogidos en este pecio ${ }^{3}$ podría indicar también que las oil bottle se encontraban incluidas en un cargamento más heterogéneo de mercancías de lujo.

2. La única oil bottle de la fase Fonteta I no se ha podido atribuir a una producción concreta (v. González Prats, 2014b: tabla 1).

3. Aparte de las bien conocidas defensas de elefante (Mederos Martín y Ruiz Cabrero, 2004) se han recogido también marfiles trabajados, peines de madera, vasos de alabastro, varios elementos de bronce (thymiaterion, caldero, piezas de muebles, el brazo de una estatueta...) y un altar de piedra caliza (Polzer, 2014). 


\subsubsection{Ibiza}

Por último, con respecto al ámbito ibicenco cabría realizar una puntualización más relativa al material recogido en el yacimiento de Sa Caleta (Ramon Torres, 2007). En su síntesis, Orsingher (2010: 51) recoge cuatro oil bottle provenientes de este yacimiento, aunque en la monografía dedicada al yacimiento se haga referencia a hasta diez posibles contenedores del tipo en cuestión (Ramon Torres, 2007: 51-52, 70, 73, 82).

Parece interesante señalar además que en este conjunto se aprecia una notable preponderancia de producciones orientales: los grupos productivos P8 y P9 definidos para el yacimiento e identificados con producciones de talleres del Mediterráneo oriental suman un total de ocho contenedores de diez (Ramon Torres, 2007), demostrando la fuerte presencia de productos con aquella procedencia a lo largo del siglo vir a.n.e. (sin embargo, v. infra).

\subsection{El perfume en el ámbito de los intercambios con las comunidades locales: nuevas oil bottles en ambientes indígenas}

Aunque menos numerosas, las novedades de los últimos años relativas a la presencia de oil bottles en ambientes indígenas peninsulares merecen también un repaso en este contexto, no solo por ampliar en cierto modo la geografía de la difusión de estos contenedores (y de sus contenidos), sino también por ahondar en un patrón de consumo muy específico y diferenciado, propio de las comunidades locales.

A este respecto, las principales novedades se refieren al sur del actual territorio portugués. En esta región se conocía ya cierto número de oil bottles procedentes de dos yacimientos con una fuerte impronta colonial fenicia: Tavira (Maia, 2000; VV.AA., 2003) y Abul A (Alcácer do Sal) (Mayet y Silva, 2000: 52). En cambio, en los últimos años se han dado a conocer tres nuevos ejemplares, todos procedentes de contextos que, a pesar de presentar evidencias muy claras de abertura al ámbito mediterráneo y más específicamente fenicio, deben en todos los casos considerarse eminentemente indígenas.

En primer lugar cabría referir una oil bottle procedente de la necrópolis de Olival do Senhor dos Mártires (Alcácer do Sal) (Gomes, 2015). El contexto de esta pieza — seguramente funerario- es desconocido, pero sus rasgos morfológicos relativamente evolucionados (cuerpo piriforme incipiente, fondo apuntado) (fig. 2, n. ${ }^{\circ}$ 27) permiten fecharla a finales del siglo VII o inicios del vi a.n.e.; es decir, a finales de la fase Ia de la necrópolis (cf. Gomes, 2016).

Este período, que coincide con el uso del cercano santuario-emporio de Abul A (Mayet y Silva, 2000), se caracteriza por cierta diversidad de soluciones funerarias, posiblemente como reflejo - al menos en parte- de la presencia de grupos exógenos y por una síntesis en cuanto a las prácticas y discursos funerarios entre elementos locales y aportaciones «orientalizantes» (cf. Gomes, 2016). 
En este marco cultural, la adopción de sustancias aromáticas en los rituales funerarios no es en absoluto inusual y puede interpretarse como un aspecto más de la apropiación local de determinados elementos de prestigio y de las prácticas que se les asocian en el contexto de la construcción de discursos de poder y representación plasmados en el ámbito de la muerte (Mayet y Silva, 2000).

Hoy por hoy, la atribución a un eventual taller gaditano que en su día propuse para esta pieza (Gomes, 2015: 301) no me parece del todo clara, aunque tampoco disponga de datos fidedignos para asignar esta pieza a un centro productor concreto. Sus características macroscópicas podrían aproximarse a los centros productores de la costa malagueña, aunque tampoco se pueda descartar a priori una proveniencia del Mediterráneo central. Por ende, y hasta que se realicen análisis más concluyentes sobre este ejemplar, la cuestión de su origen debe mantenerse con reservas.

Los otros dos ejemplares publicados recientemente provienen también de contextos funerarios, aunque en este caso del interior del territorio del Bajo Alentejo. En esta región, los trabajos preventivos realizados en el marco de la construcción de la red de irrigación del embalse de Alqueva han resultado en la identificación de un gran número de necrópolis de la I Edad del Hierro (cf. contribuciones en Jiménez Ávila, 2017).

Estos nuevos yacimientos conforman un nuevo grupo funerario muy homogéneo y con una identidad propia muy marcada, caracterizada entre otros aspectos por la riqueza y diversidad de los ajuares que acompañaban a los difuntos y que incluyen un número sustancial de elementos de prestigio exógenos, entre los cuales se cuentan los contenedores que nos ocupan.

El primero de estos se ha recogido en la necrópolis de Palhais (Beringel, Beja) (Santos et al., 2009, 2017: fig. 17), lamentablemente en posición secundaria, si bien su contexto parece indiscutiblemente funerario. Tanto la cronología general de la necrópolis como su morfología piriforme con fondo apuntado dotado de un pequeño omphalos (fig. 2, n. ${ }^{\circ} 28$ ) permiten fecharlo en la segunda mitad del siglo vi a.n.e. (Santos et al., 2017: 257-258).

La segunda pieza proveniente de estas nuevas necrópolis del interior del Alentejo se ha recuperado en la tumba 3 de la necrópolis de Carlota (São Brissos, Beja), una inhumación femenina contenida en una tumba excavada en la roca y enmarcada por un recinto delimitado por un foso igualmente excavado en el sustrato geológico (Salvador Mateos y Pereira, 2017: 340-341).

La oil bottle, que constituía el único elemento de ajuar de la tumba, corresponde a una tipología algo más arcaizante que las piezas de Alcácer do Sal y de Palhais, al presentar un cuerpo todavía relativamente globular y, sobre todo, un fondo plano delimitado por un incipiente pie de anillo (Salvador Mateos y Pereira, 2017: fig. 5, n. ${ }^{\circ} 4$ ) (fig. 2, n. ${ }^{\circ} 29$ ). Esto podría sugerir una cronología de finales del siglo vII a.n.e. o más probablemente, dado el contexto general de la necrópolis, de inicios del vi a.n.e.

Lamentablemente, no contamos con datos definitivos de cara a la identificación de los centros productores de estos contenedores del interior alentejano. La fotografía publicada de la pieza de Palhais (Santos et al., 2009: fig. 15) permite observar que esta presenta una 


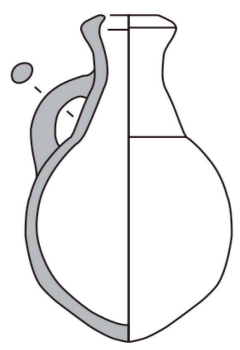

27

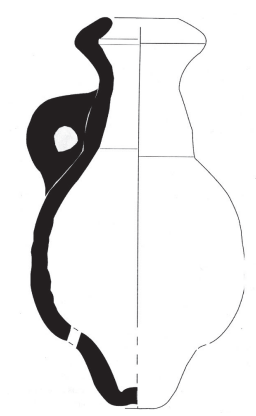

28

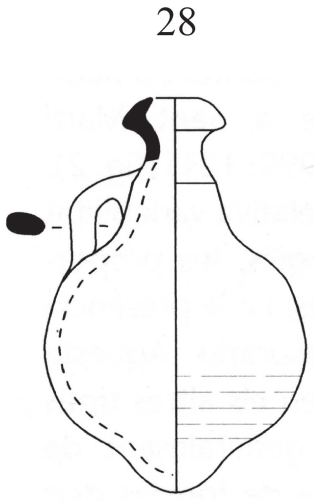

30

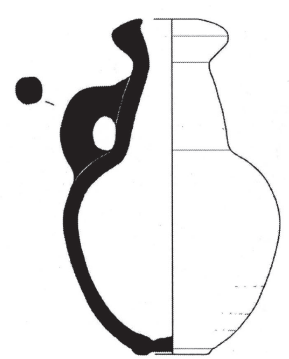

29

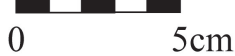

Figura 2. Oil bottles procedentes de contextos indígenas (hallazgos recientes): 1. Olival do Senhor dos Mártires (Alcácer do Sal) (seg. Gomes, 2015); 2. Palhais (Beja) (seg. Santos et al., 2017); 3. Carlota (Beja) (seg. Salvador Mateos y Pereira, 2017); 4. Vilanera (L'Escala) (seg. Aquilué et al., 2008).

pasta beige clara que recuerda las producciones de cerámica común gaditana, mientras que la de Carlota se describe como "... de cerâmica clara..." (Salvador Mateos y Pereira, 2017: 340), por lo que podría presentar características similares.

Estos datos limitados permiten además aproximarlas, aunque con reservas, a las piezas de Abul A (Mayet y Silva, 2000: 52) y a las de Tavira (Maia, 2000; VV.AA., 2003), para las que contamos con descripciones de pastas similares. Todo ello podría llevar a pensar que la difusión de las oil bottles y de sus contenidos en el actual territorio portugués se opera principalmente a partir de un centro productor concreto, ya sea la propia Gadir como propuse en otro lugar (Gomes, 2015: 301) o algún otro taller por identificar en el futuro mediante análisis más concluyentes.

El descubrimiento de estas dos piezas permite hoy ampliar la geografía de la distribución de las oil bottles en el interior portugués, evidenciando la más que probable existencia de redes regionales de (re)distribución responsables de la difusión de estos elementos 
exóticos más allá de los ambientes litorales directamente alcanzados por la presencia y el comercio oriental. Dichos hallazgos también evidencian la existencia en el territorio portugués de una dicotomía en lo relativo a las pautas de consumo entre el ámbito fenicio, donde los usos de las oil bottles y de sus contenidos son más diversos, y el ámbito indígena, donde el uso funerario es hasta el momento exclusivo.

Aparte de estos ejemplos portugueses, la única oil bottle no recogida por Orsingher que se puede señalar en este contexto proviene del otro extremo de la red comercial fenicia peninsular, en concreto de la necrópolis de Vilanera (L'Escala, Alt Empordà) (Aquilué et al., 2008, 2012). En la tumba 9 de esta necrópolis se ha recuperado un contenedor del tipo que aquí nos ocupa (Aquilué et al., 2008: fig. 8, n. ${ }^{\circ} 2$ ) (fig. 2, n. ${ }^{\circ} 30$ ) asociado a un conjunto de otros materiales de tipología fenicia (pithoi y morteros trípodes) que pueden fecharse a finales del siglo vir a.n.e., así como a un conjunto de materiales de tipología local.

Aparte de poder sumarse al inventario de elementos relacionados con el comercio fenicio documentados en territorio catalán a lo largo de las últimas décadas (v. Asensio i Vilaró, 2005; Aquilué et al., 2008; Graells i Fabregat, 2010), esta pieza refuerza el peso del uso funerario de las sustancias aromáticas contenidas en estas oil bottles también entre las comunidades del nordeste peninsular, ya señalado respecto a la pieza de la tumba X de Mas de Mussols (Maluquer, 1984: fig. 11).

\section{Perspectivas de investigación: las oil bottles en el marco del comercio de perfumes mediterráneos en la Edad del Hierro peninsular}

Sumados los hallazgos más recientes comentados en las páginas anteriores al inventario establecido en su día por Orsingher (2010), el cómputo general de las oil bottles documentadas en el territorio peninsular asciende a unos ciento ochenta individuos. Aunque no sea una cifra demasiado elevada, este número permite apreciar el peso relativamente importante de estos contenedores y sus contenidos en el marco del comercio fenicio de bienes de prestigio.

Por otra parte, la base de datos de la que hoy disponemos, a pesar de adolecer todavía de serios problemas como el conocimiento incompleto de muchos contextos arqueológicos o la falta de buenos análisis tecnológicos, permite empezar a buscar algunos patrones generales de difusión y consumo de estas oil bottles y de sus contenidos.

Hoy contamos con una reveladora cartografía de la distribución de estas piezas (fig. 3) donde se aprecia con claridad el peso de las áreas de colonización fenicia, por un lado, y, por otro, del hinterland indígena de las mismas. De hecho, la penetración de estos contenedores hacia territorios más interiores parece un fenómeno puntual posiblemente relacionado en algunos casos (p. ej., el sur interior portugués) con redes secundarias subregionales de (re)distribución. 


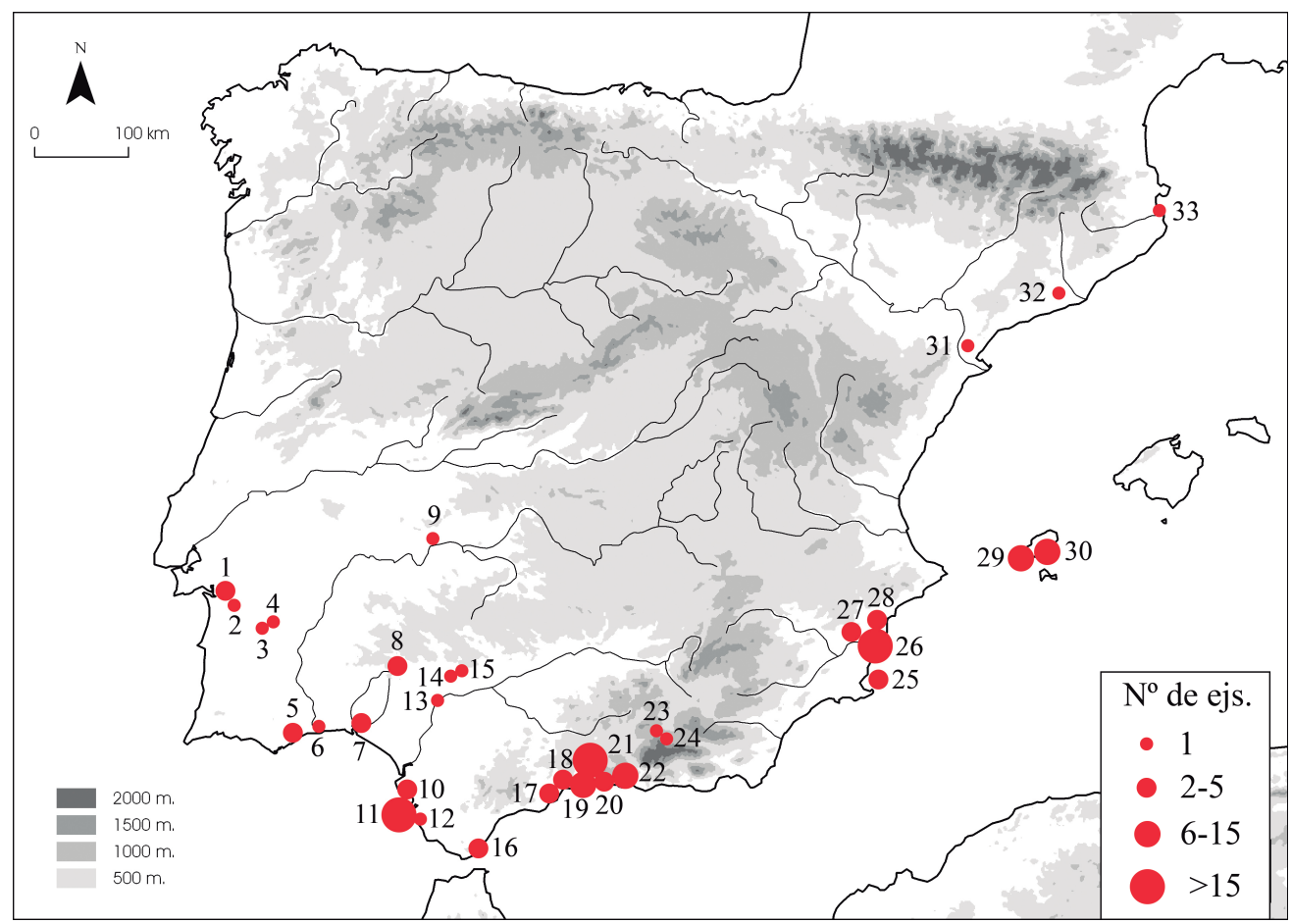

Figura 3. Distribución de las oil bottles en el territorio peninsular: 1. Abul A (Alcácer do Sal); 2. Olival do Senhor dos Mártires (Alcácer do Sal); 3. Palhais (Beja); 4. Carlota (Beja); 5. Tavira; 6. Ayamonte; 7. Huelva; 8. Cerro Salomón (Huelva); 9. Necrópolis de Medellín (Badajoz); 10. Castillo de Doña Blanca (Puerto de Santa María); 11. Cádiz; 12. Cerro del Castillo de Chiclana (Cádiz); 13. Carambolo Bajo (Sevilla); 14. Carmona; 15. Cruz del Negro (Carmona); 16. Gorham's Cave (Gibraltar); 17. Cerro del Villar (Málaga); 18. Málaga; 19. Morro de Mezquitilla (Málaga); 20. Toscanos (Málaga); 21. La Pancha (Málaga); 22. Chorreras (Málaga); 23. Cerro de la Mora (Granada); 24. Cerro de los Infantes (Granada); 25. Bajo de la Campana (Cartagena); 26. La Fonteta (Alicante); 27. Peña Negra (Alicante); 28. Saladares (Alicante); 29. Puig des Molins (Ibiza); 30. Sa Caleta (Ibiza); 31. Mas de Mussols (Tarragona); 32. Turó de la Font de la Canya (Barcelona); 33. Vilanera (Alt Empordà).

Por otra parte, y en términos estrictamente cuantitativos, algunos centros destacan en cuanto al volumen de hallazgos y podrían interpretarse como centros preferenciales de consumo, así como plausiblemente de distribución de estos contenedores. Este parece ser el caso de La Fonteta (González Prats, 2014b) y también de Cádiz (Córdoba Alonso y Ruiz Mata, 2005; Torres Ortiz et al., 2014; Maya Torcelly et al., 2014; Belizón Aragón et al., 2014; Sáez Romero e Higueras-Milena Castellano, 2016) y de Ibiza (Ramon Torres, 1982: 36; v. tb. Orsingher, 2010). A estos núcleos cabría añadir el asentamiento colonial de Toscanos (Vélez-Málaga) en cuyo territorio la producción de este tipo de contenedores parece bien atestiguada (Martín Córdoba et al., 2006: 271).

El hecho de que se conozca relativamente bien la evolución tipológica de estos contenedores, así como la existencia de un número cada vez mayor de piezas procedentes 


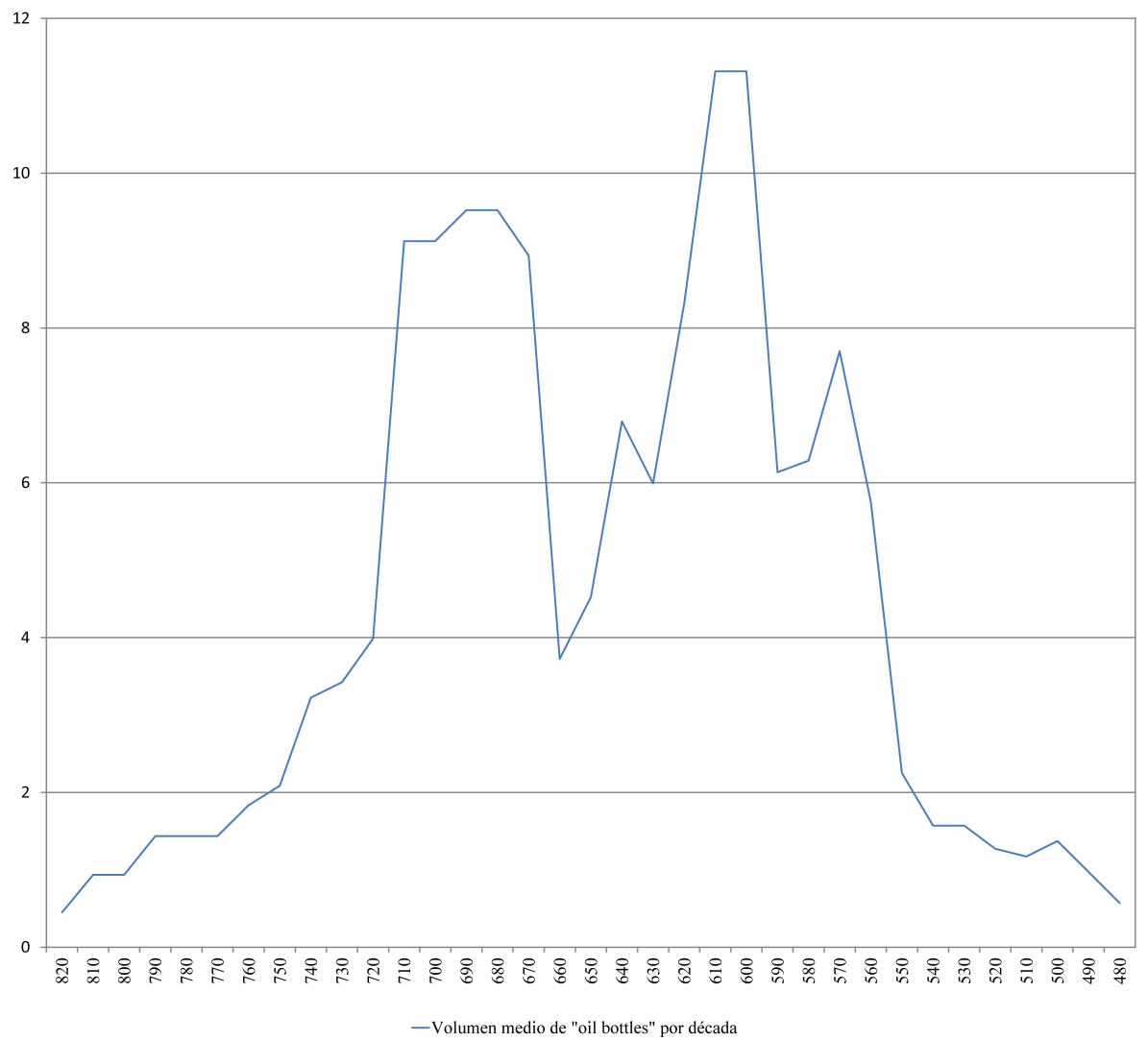

Figura 4. Evolución diacrónica de la difusión/consumo de las oil bottles fenicias y sus contenidos (volumen medio por década). No se incluyen las piezas del centro alfarero de La Pancha, ya que no habrán llegado a entrar en circulación.

de contextos bien fechados, permite establecer una primera aproximación a la evolución diacrónica del volumen global del comercio de las sustancias aromáticas contenidas en las oil bottles (fig. 4).

A pesar de todos los condicionantes de la muestra, el análisis de dicha evolución ofrece varios puntos de interés: cabría señalar el incremento paulatino de las oil bottles en los primeros momentos de la presencia fenicia en la península ibérica, que solo dará lugar a una auténtica explosión con la consolidación de dicha presencia y de las relaciones de los colonos fenicios con las comunidades locales a finales del siglo viII a.n.e..

A partir de dicha fecha, la demanda de las sustancias aromáticas contenidas en estas oil bottles se ha mantenido siempre a niveles relativamente elevados, aunque se aprecie una aparente quiebra a mediados del siglo vII a.n.e. cuyo significado no es fácil de interpretar. 
Sin embargo, este episodio podría relacionarse con el ascenso de nuevos centros productores y con los consecuentes ajustes relativos a los respectivos mercados.

Tras dicho episodio se aprecia una reanudación en el consumo de estos contenedores y de sus contenidos, que alcanza a finales del siglo vir-inicios del vi a.n.e. su momento de mayor popularidad antes de sufrir una nueva y esta vez permanente quiebra a mediados de siglo, relacionada sin duda con la reestructuración del mundo fenicio occidental a raíz de la llamada "crisis del siglo vi», y que precede a la desaparición de estos contenedores hacia el tercer cuarto de siglo (Orsingher, 2010: 57).

Si los datos disponibles permiten, reconstruir a grandes rasgos la historia interna del comercio de estas oil bottles y de sus contenidos en el extremo Occidente, el peso relativo de los distintos centros productores en el marco de ese comercio es hoy por hoy mucho más difícil de valorar. Como puede apreciarse en el gráfico de la figura 5a, la vasta mayoría de los contenedores conocidos no se ha podido atribuir hasta el momento a un centro productor concreto.

Así, y a pesar de los nuevos datos aportados por los trabajos reseñados en las páginas anteriores, el análisis del peso de los distintos centros productores en el aprovisionamiento del territorio peninsular sigue siendo un ejercicio muy arriesgado. En todo caso, en el gráfico de la figura 5b se ha intentado plasmar los datos que sí están disponibles, aunque las limitaciones de la información se hagan todavía más evidentes al representarla gráficamente.

No obstante, sí se aprecian en ese gráfico algunas tendencias que, al correlacionarse de manera directa con los datos aportados por los análisis ya comentados, podrían considerarse pertinentes. Señálese, por ejemplo, la aparente existencia de una fase inicial donde el predominio de las importaciones orientales es absoluto, y que parece congruente con lo que sería esperable en la fase inicial de la expansión colonial fenicia. Igual de sugerente es la rápida aparición de producciones peninsulares que parecen disputarse el mercado con los productos orientales desde la primera mitad del siglo viII a.n.e..

Por otra parte, es interesante notar el aparente retroceso de la "cuota de mercado" de los productos orientales en torno a mediados del siglo vir a.n.e. Este fenómeno podría, con las debidas reservas, relacionarse con la ya comentada quiebra en la circulación global de las oil bottles que se aprecia precisamente en estos momentos, lo cual llevaría a pensar que se asistió entonces a un reajuste de las redes de abastecimiento de sustancias aromáticas a los mercados peninsulares.

Es además interesante notar que que parece ser ese el momento en que los productos del Mediterráneo central se afianzan en los mercados occidentales, quizá como resultado de ese mismo proceso de reajuste cuya naturaleza cabría seguir explorando en el futuro con una base de datos más sólida.

De todos modos, estas consideraciones deben tomarse con mucha cautela por las razones ya explicadas, y se necesitan datos mucho más sólidos antes de entrar a valorar las posibles dinámicas comerciales que hay detrás de los perfiles de consumo de cada región en cuanto a sus respectivos centros de abastecimiento. 

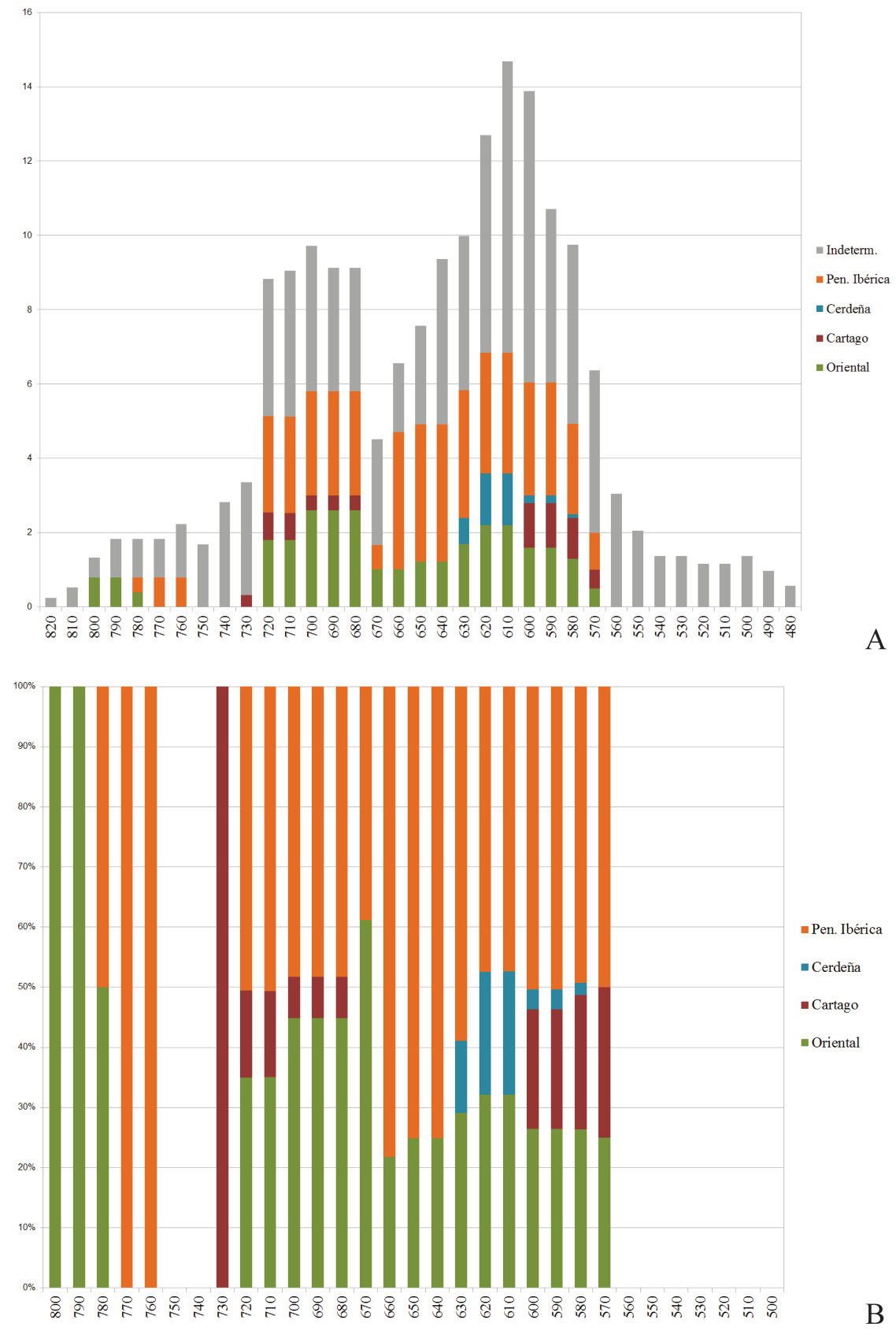

Figura 5. Evolución diacrónica de la representatividad de los varios centros productores de oil bottles actualmente identificados en la península ibérica: A. valores absolutos (incluyen piezas no atribuidas); B. valores relativos (solo piezas atribuidas). 

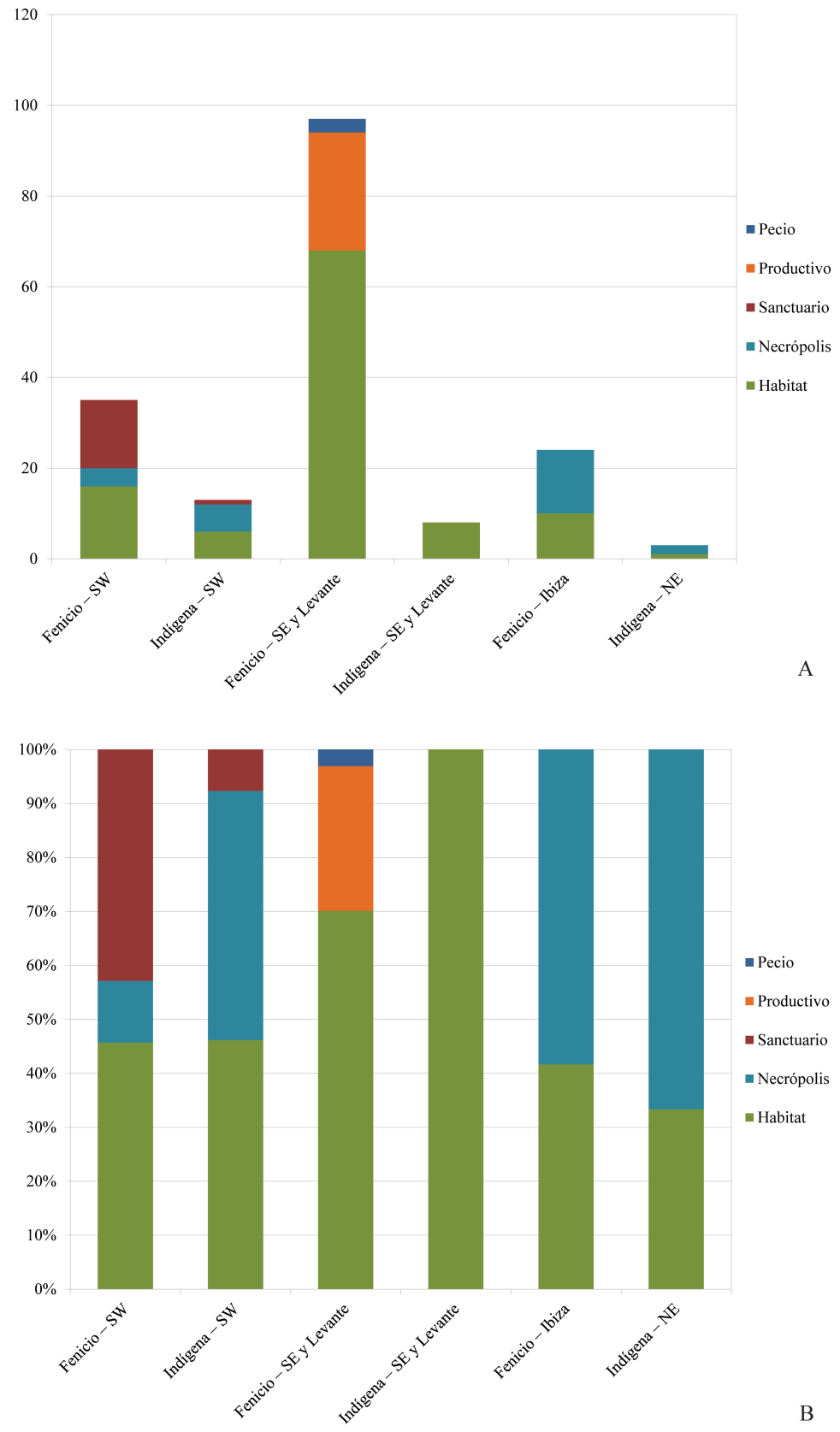

Figura 6. Repartición de las oil bottles conocidas por contextos funcionales en los distintos grandes bloques culturales y geográficos considerados. 
Las pautas de consumo pueden, sin embargo, valorarse desde otro punto de vista: el de los contextos funcionales de uso de las sustancias aromáticas contenidas en estas vasijas, que pueden acercarnos a una valoración de las prácticas en que se empleaban, así como de los significados sociales que pueden haber tenido en distintos ámbitos culturales.

En el gráfico de la figura 6 se presenta la repartición de las oil bottles peninsulares según los diversos contextos funcionales de uso, desglosados además en ámbitos culturales y geográficos genéricos (comunidades fenicias del Suroeste, del Sureste/Levante y de Ibiza, por un lado, y comunidades indígenas del Suroeste, del Sureste/Levante y del Nordeste, por otro).

A pesar de la existencia de solapamientos esperables, los resultados de esa repartición sí parecen indicar pautas sociales de consumo algo diferenciadas de comunidad a comunidad y de región a región.

Así, entre las comunidades fenicias del Atlántico se aprecian unas pautas de consumo más diversificadas que entre sus homólogas de la costa mediterránea andaluza y levantina, con una presencia sustancial de oil bottles en putativos ambientes religiosos, y en menor medida funerarios, que es totalmente desconocida en las colonias del litoral andaluz y alicantino. Igualmente distinta es la situación en Ibiza, donde el peso de la documentación funeraria es predominante.

Las comunidades indígenas del suroeste peninsular presentan igualmente unas pautas de consumo más diversificadas. En este ámbito, el uso funerario del perfume tiene un peso significativo, al parecer equiparable al de su uso en contextos de lo cotidiano, tal y como parece ocurrir entre las comunidades del nordeste peninsular. Los escasos registros de oil bottles en ambientes indígenas del sureste y Levante peninsular, por contra, se refieren exclusivamente a ambientes domésticos, reflejando así de alguna manera el propio uso que se hacía de estos contenedores en los ámbitos coloniales regionales.

Ahora bien, si la diversidad de contextos sociales de uso de las sustancias transportadas en estos pequeños contenedores se puede apreciar incluso en una lectura general y aglutinadora como la que aquí he presentado, no cabe duda de que dichas sustancias han desempeñado papeles sociales muy diversos en las distintas comunidades que las consumieron. Sin embargo, para seguir desentrañando los significados atribuidos a estos perfumes por cada comunidad específica, es necesario un abordaje contextual más fino de todo el entorno material de estos contenedores, trabajo que transciende los objetivos de esta contribución.

Lo que sí parece demostrado es la importancia que estas oil bottles y sus contenidos han tenido en la introducción en el ámbito peninsular del uso del perfume. De hecho, y a pesar de ser más comunes en ambientes coloniales, estos contenedores han conocido una difusión mucho más amplia en el ámbito indígena que otros posibles contenedores de perfume contemporáneos, como por ejemplo los pequeños vasos de alabastro (López Castro, 2006; Pellicer Catalán, 2007). 
No parece además casual que la desaparición de estos contenedores coincida en el tiempo con la aparición en los mercados occidentales de un conjunto variopinto de contenedores de perfumes, como los aryballoi y alasbastra corintios, naucratitas y, en menor medida, de la Grecia de Leste ( $c f$. Rouillard, 1991; Domínguez Monedero y Sánchez Fernández, 2000; Jiménez Ávila y Ortega Blanco, 2004) y, más tarde, los pequeños contenedores de vidrio formados sobre núcleo de arena que acabarán inundando los mercados peninsulares a lo largo del siglo v e inicios del Iv a.n.e. (Feugère, 1989; Jiménez Ávila, 1999, 2003).

El consumo de los contenidos de las oil bottles fenicias parece de hecho haber introducido entre las comunidades peninsulares una costumbre de uso de sustancias aromáticas que perduró más allá de la vigencia de estos contenedores y que ha seguido evolucionando y progresando a lo largo de todo el I milenio a.n.e. acompañando las mutaciones sociopolíticas regionales y las transformaciones geopolíticas que durante ese período se suceden en el Mediterráneo ( $c f$. López Rosendo, 2005).

Al terminar esta breve puesta al día sobre las oil bottles fenicias de la península ibérica, no puede obviarse lo mucho que queda por hacer para obtener de estos contenedores todo su potencial informativo. Se ha insistido mucho, por ejemplo, en la necesidad de averiguar su procedencia recurriendo a metodologías analíticas más seguras.

Sin embargo, la realización de análisis de contenidos debe considerarse tan o más importante que ese estudio de la estructura física de las piezas mismas. Tras décadas estudiando estas oil bottles, seguimos sin saber qué tipo de sustancia contenían exactamente, ni si los perfumes o aceites perfumados que presumiblemente transportaban seguían recetas más o menos estandarizadas.

No sabemos tampoco si los varios talleres productores que poco a poco se van definiendo gracias a la investigación más reciente seguían recetas similares, o si la producción de sustancias aromáticas capitalizó en cada ambiente colonial los recursos naturales locales, utilizando aromata diferenciados. Cuando analizamos la variación diacrónica del peso de los distintos centros productores, por ejemplo, ¿estamos tratando únicamente cuestiones de logística del aprovisionamiento, o más bien cambios reales en el gusto de las comunidades consumidoras que eligen entre productos y tradiciones de perfumería distintas?

Esto solo podrá abordarse en el futuro mediante protocolos de investigación interdisciplinarios y la aplicación de técnicas analíticas cada vez más sofisticadas en el estudio de estos y de otros contenedores de perfumes y sustancias aromáticas. Solo así se podrá explorar en profundidad el potencial informativo de estos elementos para una comprensión más profunda de las interconexiones que caracterizaron el Mediterráneo antiguo. 


\section{Adenda}

Cuando este trabajo se encontraba ya terminado se publicó la monografía de las excavaciones en la necrópolis fenicia de Ayamonte (Huelva) (Marzoli y García Teyssandier, 2018) en la que se dio a conocer una segunda oil bottle procedente de la tumba 8, excavada en la campaña de 2013 (Marzoli y García Teyssandier, 2018: 170-171, figs. 143a, 144a y 145a) que acompañaba una cremación en una urna «Cruz del Negro» fechada en la segunda mitad del siglo vIII o inicios del siglo vir a.n.e. (Marzoli y García Teyssandier, 2018: 171).

Esta pieza, que ha sido objeto de análisis por activación de neutrones, corresponde a una producción de un taller de la región de Cádiz (Marzoli y García Teyssandier, 2018). Se han realizado además, y por primera vez, análisis químicos para determinar los contenidos de este ejemplar, cuyos resultados sin embargo han sido negativos (Marzoli y García Teyssandier, 2018), lo que parece indicar que esta pieza o bien nunca se utilizó como contenedor o se usó para contener una sustancia neutra desde el punto de vista químico.

\section{Bibliografía}

AQUILUÉ, X., CASTANYER, P., SANTOS, M. y TREMOLEDA, J, 2008, Noves evidències del comerç fenici amb les comunitats indígenes de l'entorn d'Empúries, en D. GARCIA i RUBERT, I. MORENO MARTÍNEZ Y F. GRACIA ALONSO (coords.), Contactes. Indígenes i fenicis a la Mediterrània occidental entre el segles VIII $i$ VI ane, Ajuntament d'Alcanar, Alcanar, 171-190.

AQUILUÉ, X., CASTANYER, P., SANTOS, M. y TREMOLLEDA, J., 2012, El paisatge funerari en el territori d'Empúries, entre el Bronze Final i la Primera Edat del Ferro, en M. C. ROVIRA HORTALÀ, F. J. LÓPEZ CACHERO Y F. MAZIÈRE (dirs.), Les necròpolis d'incineració entre l'Ebre $i$ el Tíber (segles IX-VI aC): metodologia, pràctiques funeràries i societat, Museu d'Arqueologia de Catalunya, Barcelona, 75-90.
ASENSIO i VILARÓ, D., 2005, La incidencia fenicia entre las comunidades indígenas de la costa catalana (siglos VII-VI AC): ¿un fenómeno orientalizante?, en S. CELESTINO PÉREZ y J. JIMÉNEZ ÁVILA (eds.), El Período Orientalizante, CSIC, Madrid, 551-564.

BELIZÓN ARAGÓN, R., BOTTO, M. y LEGUPÍN TUBÍO, I., 2014, Conjunto funerario fenicio en el extremo sureste de la necrópolis de Gadir, en M. BOTTO (ed.), Los Fenicios en la Bahía de Cádiz, Fabrizio Serra, Roma, 202-224.

BISI, A. M. ${ }^{a}$, 1970, La ceramica punica. Aspetti e problemi, L'Arte Tipografica, Nápoles.

BOTTO, M. (ed.), 2014, Los Fenicios en la Bahía de Cádiz, Fabrizio Serra, Roma. 
BUENO SERRANO, P., 2014, Un asentamiento del Bronce Final - Hierro I en el Cerro del Castillo, Chiclana, Cádiz. Nuevos datos para la interpretación de Gadeira, en M. BOTTO (ed.), Los Fenicios en la Bahía de Cádiz, Fabrizio Serra, Roma, 225-251.

CÓRDOBA ALONSO, I. y RUIZ MATA, D., 2005, El asentamiento fenicio arcaico de la calle Cánovas del Castillo (Cádiz): un análisis preliminar, en S. CELESTINO PÉREZ

y J. JIMÉNEZ ÁVILA (eds.), El Período

Orientalizante, CSIC, Madrid, 1269-1322.

CULICAN, W., 1970, Phoenician Oil Bottles and Tripod Bowls, Berytus XIX, 5-18.

DOMINGUEZ MONEDERO, A. y SÁNCHEZ FERNÁNDEZ, C., 2001, Greek Pottery from the Iberian Peninsula. Archaic and Classical Periods, Brill, Leiden.

FEUGÈRE, M., 1989, Les vases en verre sur noyau d'argile en Méditerranée nord-occidentale, en M. FEUGÈRE (ed.), Le verre préromain en Europe occidentale, Éditions Monique Mergoil, Montpellier, 29-62.

GARCÍA TEYSSANDIER, E. y MARZOLI, D., 2013, Phönizische Gräber in Ayamonte (Huelva, Spanien): ein Vorbericht, Madrider Mitteilungen 54, 89-158.

GENER BASALLOTE, J. M. ${ }^{a}$, NAVARRO GARCÍA, M. Á., PAJUELO SÁEZ, J.-M., TORRES ORTIZ, M. y LÓPEZ ROSENDO, E., 2014, Arquitectura y urbanismo de la Gadir fenicia: el yacimiento del «Teatro Cómico» de Cádiz, en M. BOTTO (ed.), Los Fenicios en la Bahía de Cádiz, Fabrizio Serra, Roma, 14-50.

GOMES, F. B., 2015, As "oil bottles" de tipo fenício no território português e o consumo de substâncias aromáticas, O Arqueólogo Português V:3, 289-310.

GOMES, F. B., 2016, Contactos culturais e discursos identitários na I Idade do Ferro do Sul de Portugal (sécs. VIII-V a.n.e.): leituras a partir do registo funerário (tesis doctoral presentada en la Universidad de Lisboa, inédita).

GONZÁLEZ PRATS, A., 1982, Ampollas de perfume fenicias en el horizonte orientalizante de la Sierra de Crevillente, Helike 1, 139-143.
GONZÁLEZ PRATS, A. (coord. y ed.), 2014a, La Fonteta-2. Estudio de los materiales arqueológicos hallados en la colonia fenicia de la actual desembocadura del río Segura (Guardamar, Alicante), Universitat d'Alacant, Alacant.

GONZÁLEZ PRATS, A., 2014b, Tipo 9 y Tipo 10. Frascos con asa realzada y Ampollas con estrecho gollete, en A. GONZÁLEZ PRATS (coord. y ed.), La Fonteta-2. Estudio de los materiales arqueológicos hallados en la colonia fenicia de la actual desembocadura del río Segura (Guardamar, Alicante), Universitat d'Alacant, Alacant, 420-442.

GRAELLS i FABREGAT, R., 2010, Las tumbas con importaciones y la recepción del Mediterráneo en el Nordeste de la Península Ibérica (siglos VII-VI aC.), Universitat de Lleida, Lleida.

JIMÉNEZ ÁVILA, J., 1999, Los objetos de vidrio procedentes del yacimiento de Pajares: estudio preliminar, en S. CELESTINO PÉREZ (ed.), El Yacimiento Protohistórico de Pajares. Villanueva de La Vera. Cáceres. 1. Las necrópolis y el Tesoro Áureo, Junta de Extremadura, Cáceres, 139-153.

JIMÉNEZ ÁVILA, J., 2003, Los objetos de pasta vítrea de Cancho Roano, en S. CELESTINO PÉREZ (ed.), Cancho Roano VIII. Los materiales arqueológicos, Junta de Extremadura, Cáceres, 261-291.

JIMÉNEZ ÁVILA, J. y ORTEGA BLANCO, J., 2004, La cerámica griega en Extremadura, Museo Nacional de Arte Romano, Mérida.

JIMÉNEZ ÁVILA, J. (ed.), 2017), Sidereum Ana III. El Río Guadiana y Tartessos, Consorcio Ciudad Monumental Histórico-Artística e Arqueológica de Mérida, Mérida.

LÓPEZ CASTRO, J. L., 2006, Colonials, merchants and alabaster vases: the western Phoenician aristocracy, Antiquity 80, 74-88.

LÓPEZ ROSENDO, E., 2005, El perfume en los rituales orientalizantes de la península Ibérica, en S. CELESTINO PÉREZ y J. JIMÉNEZ ÁVILA, J. (eds.), El Período Orientalizante, CSIC, Madrid, 669-682.

MAASS-LINDEMANN, G., 1999, La cerámica de las primeras fases de la colonización fenicia en España, en A. GONZÁLEZ PRATS (ed.), La 
cerámica fenicia en Occidente: centros de producción y áreas de comercio. Actas del I Seminario Internacional sobre Temas Fenicios, Instituto Alicantino Juan GilAlbert, Alacant, 129-148.

MAIA, M. a , 2000, Tavira fenícia. O território para Ocidente do Guadiana, nos inícios do I milénio a.C., en A. GONZÁLEZ PRATS (ed.), Fenicios y Territorio. Actas del II Seminario Internacional sobre Temas Fenícios, Instituto Alicantino Juan GilAlbert, Alacant, 121-150.

MALUQUER DE MOTES, J., 1984, La necrópolis paleoibérica de "Mas de Mussols", Tortosa (Tarragona), CSIC/Universidad de Barcelona, Barcelona.

MARTÍN CÓRDOBA, E., RAMÍREZ SÁNCHEZ, J. de D. y RECIO RUIZ, Á., 2006, Producción alfarera fenicio-púnica en la costa de VélezMálaga, Mainake XXVIII, 257-287.

MARZOLI, D. y GARCÍA TEYSSANDIER, E., 2018, Die Phönizische Nekropole von Ayamonte, Deutsches Archäologisches Institut/Harrassowitz Verlag, Wiesbaden.

MAYA TORCELLY, R., JURADO FRESNADILLO, G., PAJUELO SÁEZ, J.-M. Y TORRES ORTIZ, M. (2014), Nuevos datos sobre la posible ubicación del Kronion de Gadir: las evidencias de época fenicia arcaica, en M. BOTTO (ed.), Los Fenicios en la Bahía de Cádiz, Fabrizio Serra, Roma, 156-180.

MAYET, F. y SILVA, C. T. DA, 2000, L'établissement phénicien d'Abul. Portugal, Diffusion du Boccard, París.

MEDEROS MARTÍN, A. y RUIZ CABRERO, L. A., 2004, El pecio fenicio del Bajo de la Campana (Murcia, España) y el comercio del marfil norteafricano, Zephyrus 57, 263-281.

ORSINGHER, A., 2010, Le oil bottle fenicie: analisi dei contesti e considerazione crono-tipologiche, Sardinia, Corsica et Baleares Antiquae VIII, 37-69.

PELLICER CATALÁN, M., 2007, La Necrópolis Laurita (Almuñécar, Granada) en el contexto de la colonización fenicia, Universitat Pompeu Fabra, Barcelona.

POLZER, M. E., 2014, The Bajo de la Campana Shipwreck and Colonial Trade in Phoenician
Spain, en J. ARUZ, S. B. GRAFF e Y. RAKIC, Y. (eds.), From Assyria to Iberia at the Dawn of the Classical Age, Metropolitan Museum of Art, Nueva York, 230-242.

RAMON TORRES, J., 1982, Cuestiones de comercio arcaico: frascos de aceite perfumado en el Mediterráneo central y occidental, Ampurias 44, 17-41.

RAMON TORRES, J., 2007, Excavaciones arqueológicas en el asentamiento fenicio de Sa Caleta (Ibiza), Universitat Pompeu Fabra, Barcelona.

ROUILLARD, P., 1991, Les Grecs et la Péninsule Ibérique: du vIII au Iv siècle avant Jésus-Christ, Diffusion du Boccard, París.

ROUILLARD, P., GAILLEDRAT, E. y SALA SELLÉS, F., 2007, L'établissement protohistorique de La Fonteta : (fin viII - fin vi siècle av. J.C.), Casa de Velázquez, Madrid.

RUIZ MATA, D., PÉREZ, C. y GÓMEZ FERNÁNDEZ, V., 2014, Una nueva zona fenicia de época arcaica en Cádiz: el solar de la «calle Ancha, n²9», en M. BOTTO (ed.), Los Fenicios en la Bahía de Cádiz, Fabrizio Serra, Roma, 83-122.

SÁEZ ROMERO, A. e HIGUERAS-MILENA CASTELLANO, A., 2016, Cerámicas fenicias arcaicas de procedencia subacuática del área de la Caleta (Cádiz): ensayo de contextualización e interpretación histórica, Cuadernos de Prehistoria y Arqueología de la Universidad Autónoma de Madrid 42, 119-142.

SALVADOR MATEOS, R. y PEREIRA, J. A., 2017, A paisagem funerária a Oeste de Beja no Período Orientalizante: as necrópoles da Carlota (São Brissos) e de Cinco Réis 8 (Santiago Maior), en J. JIMÉNEZ ÁVILA (coord.), Sidereum Ana III. El Río Guadiana y Tartessos, Consorcio Ciudad Monumental Histórico-Artística e Arqueológica de Mérida, Mérida, 333-352.

SANTOS, F., ANTUNES, A. S., DEUS, M. DE y GRILO, C., 2017, A necrópole de Palhais (Beringel, Beja), en J. JIMÉNEZ ÁVILA (coord.), Sidereum Ana III. El Río Guadiana y Tartessos, Consorcio Ciudad Monumental Histórico-Artística e Arqueológica de Mérida, Mérida, 227-262. 
SANTOS, F., ANTUNES, A.S., GRILO, C. y DEUS, M. DE, 2009, A necrópole da I Idade do Ferro de Palhais (Beringel, Beja). Resultados preliminares de uma intervenção de emergência no Baixo-Alentejo, en IV Encuentro de Arqueología del Suroeste Peninsular, Universidad de Huelva, Huelva,746-804.

SCHUBART, H., 1985, El asentamiento fenicio del s. viII a.C. en el Morro de Mezquitilla, Aula Orientalis 3, 59-83.
TORRES ORTIZ, M., LÓPEZ ROSENDO, E., GENER BASALLOTE, J.M. ${ }^{a}$, NAVARRO GARCÍA, M. Á. y PAJUELO SÁEZ, J.-M., 2014, El material cerámico de los contextos fenicios del «Teatro Cómico" de Cádiz: un análisis preliminar, en M. BOTTO (ed.), Los Fenicios en la Bahía de Cádiz, Fabrizio Serra, Roma, 51-82.

VV.AA., 2003, Tavira: Território e Poder, Museu Nacional de Arqueologia, Lisboa. 
